\title{
Discussion of problems in transport logistics company supermix concrete s/a
}

\author{
Alfredo Nogueira Mariano ${ }^{1}$, Emanoel Corrêa Campos ${ }^{1}$, Sandro Oliveira Nunes ${ }^{1}$ \\ Alfredonogueira.mariano@hotmail.com,Emanoel.campos@hotmail.com, Adm-sandro@hotmail.com
}

${ }^{1,2}$ Centro Universitário do Norte (UNINORTE). Av. Joaquim Nabuco, 1469, Centro. Manaus-Amazonas-Brasil. CEP: 69005-290.

Fone: +55 (92) 32125000

\begin{abstract}
This study presents causes that directly interfere with the uptake and transport logistics company revenue Supermix Concrete S/A, from raw material collection, production and distribution of the product through the urban road network process. The aim of the paper is to identify the problems in transportation, from manufacturing the product until its delivery to the customer, measuring losses and their impacts. The methods and techniques used were: collecting data from interviews with employee's script, checklist of direct observation and documentary research. After the tabulation and analysis of these data, find the degree of traffic congestion of transportation logistics services inside and outside the company. Dare to result from calculations of production management and operations were formulated charts for better viewing of the production loss and consequently the loss of profit. The results showed that the research obtain their goals achieved, because losses were measured, identified problems arising from and around the company.
\end{abstract}

Key-words: Distribution Logistics, Transport, Logistic Problems.

\section{Discussão de problemas de logística de transorte na empresa Supermix Concreto S/A}

\section{RESUMO}

No estudo das causas que interferem directamente com as empresas de transporte e logística no volume de negócios, Supermix Concreto S/A, desde a recolha de matérias-primas, produção e distribuição do produto são apresentados através do processo da rede rodoviária Urban. O objetivo deste é para identificar os problemas no transporte, desde a fabricação até a entrega ao cliente, a medição das perdas e seus impactos. Os métodos e técnicas utilizados foram: a recolha de informações a partir de entrevistas com funcionários, lista de verificação de observação direta e pesquisa documental. Após tabulação e análise destes dados, foi determinado o grau de congestionamento de serviços de logística de transporte dentro e fora da empresa. Através dos resultados da avaliação da gestão de produção e operações para melhores gráficos de visualização de produção perdida e os lucros daí perdidos foram feitas. Os resultados mostraram que a investigação atingiu os seus objetivos.

Palavras-chave: Logística de Distribuição, Transporte, problemas logísticos.

\section{INTRODUÇÃO}

A presente pesquisa dispõe de causas que evidenciam problemas no transporte logístico da empresa Supermix Concreto S/A, problemas esses que trazem impactos financeiros significativos à organização, se minimizados ou sanados, pode-se proporcionar melhores resultados em todas as áreas envolvidas. $\mathrm{O}$ tema abrange diretamente a relação entre a logística de seu transporte com seu custo beneficio. Sabe-se que um planejamento logístico mal elaborado pode pôr em risco as metas estabelecidas e a própria sobrevivência da organização. A logística empresarial trata de todas as atividades de movimentação e armazenagem, que facilitam o fluxo de produtos desde o ponto de aquisição da matéria-prima até o ponto de consumo final, assim como dos fluxos de informações que colocam os produtos em movimento, com o propósito de providenciar níveis de serviço adequados aos clientes a um custo razoável [1].

A pesquisa justifica-se, pela necessidade de novos processos, a fim de acompanhar o ritmo de mercado da construção civil, a ponto de oferecer a seus clientes potenciais, vantagens que a torne mais competitiva. Essa vantagem contribuirá ao fortalecimento de sua marca, e seu produto, que nos dias atuais é assunto de grande relevância, já que sua permanência nesse mercado é baseada em estratégias e serem totalmente aptas a essas mudanças. Através dessa inovação, novos recursos serão utilizados em benefício de todos, clientes, fornecedores, funcionários, acionistas e a sociedade em geral, com objetivos comuns. A logística é o processo de gerenciar estrategicamente aquisição, movimentação e armazenagem de materiais e produtos acabados bem como os relativos fluxos de modo a maximizar as lucratividades presente e futura através 
da redução dos custos [2]. A problemática se da a partir do processo de armazenamento de insumos junto à linha de produção, ambos realizados ao mesmo tempo envolvendo abastecimento de linha, de almoxarifado, entrada e saída de betoneiras, armazenagem e retirada de insumos e uma única via de acesso.

Segundo [3], "a formulação do problema prende-se ao tema proposto: ela esclarece a dificuldade específica com a qual se defronta e que se pretende resolver por intermédio da pesquisa". Diante dessas vertentes, o que fazer para aperfeiçoar o processo de produção e transporte logístico da empresa Supermix Concreto S/A?

\section{REVISÃO BIBLIOGRÁFICA II.1. A EVOLUÇÃO DA LOGISTICA}

Embora a logística fosse inicialmente aplicada a operações militares, o seu impacto mais significativo está sendo sentido através das funções de produção, distribuição e consumo [4]. Carências logísticas sempre existiram, somente após a Segunda Guerra é que começaram os primeiros estudos sobre o assunto. A primeira citação de logística, utilizada no sentido de apoio à guerra, deveu-se a [5], que escreveu: "Logística é a ação que conduz à preparação e à sustentação de campanhas".

A troca de bens é uma característica constante da atividade econômica humana. Era uma vez fundamental para a ascensão da economia mercantil na Europa medieval e tornouse uma atividade de grande escala durante a revolução industrial [6]. Para [7] nos trouxe os princípios da logística moderna, o criador conceitual do fordismo. Embora suas ideias estivessem relacionadas com a melhoria continua da eficiência dentro da fábrica através da organização de tarefas do trabalhador ao longo de uma linha de montagem, as dimensões temporais introduzidas, como a sequência, duração, horário, ritmo, tempo de sincronização e perspectiva é de alta importância para a gestão logística.

\section{II.2. LOGÍSTICA NOS DIAS ATUAIS}

A organização e a tecnologia de distribuição moderna são incorporadas em um quadro macro e microeconômico de mudanças. Ele pode ser mais ou menos caracterizado pelos termos de flexibilização e globalização. Flexibilização representa, muito além da interpretação restritiva da "especialização flexível", um modo altamente diferenciada, fortemente pelo mercado e orientada para o cliente de criação de valor agregado. Produção e distribuição contemporânea já não estão sujeita a atividade de uma única empresa, mas cada vez mais praticado em redes de fornecedores e subcontratados [8][9][10].

Logística é o amplo conjunto de atividades dedicadas à transformação e circulação de bens, tais como o fornecimento de material de produção, a função de distribuição de núcleo e

\section{MATERIAIS E MÉTODOS}

O estudo de caso realizado na empresa Supermix Concreto S/A possibilitou um grande e detalhado de transporte, comércio por grosso e a retalho e também a prestação de domicílios com bens de consumo, bem como as informações relacionadas flui [11]. A logística se preocupa com o modo pelo qual a administração pode prover melhor rentabilidade nos serviços de distribuição [...], através do desenvolvimento de conceitos e métodos de planejamento, organização e controle efetivo para as atividades de movimentação e armazenagem, visando facilitar o fluxo de produtos [12]. Por ser uma área que envolve operações complexas e devido a sua particularidade geográfica característica, seu processo está sempre se renovando e a "Implementação das melhores práticas logísticas tornou-se uma das áreas mais desafiadoras e interessantes da administração nos setores privado e público" [13].

\section{II.3. DEFINIÇÕES DO ASSUNTO}

Pode-se conceituar logística como sendo a soma de quatro atividades: a de aquisição de matéria-prima, movimentação/locomoção, armazenagem e entrega de produto final. Para que todas essas atividades funcionem, é preciso que as atividades de planejamento logístico, querem sejam de materiais ou de processos. A palavra Logística, conforme o Dicionário Aurélio, tem como base o Francês Logistique e tem como uma de suas definições: Conforme [14].

A parte da arte da guerra que trata do planejamento e da realização de: projeto e desenvolvimento, obtenção, armazenamento, transporte, distribuição, reparação, manutenção e evacuação de material para fins operativos ou administrativos. De acordo com o Conselho Profissional da Gestão da Cadeia de Suprimentos, Logística é a parte da gestão da cadeia de suprimentos que planeja, [...] e controla o fluxo eficiente e eficaz [...] de mercadorias, serviços e informações relativas desde o ponto de origem até o ponto de consumo com o propósito de atender às exigências dos clientes [15].

Um dos maiores desafios que são enfrentados pelos administradores na atualidade são os custos logísticos, onde produtos e serviços devem ter qualidade e com custos competitivos. De acordo com [1], "os custos afetam o nível de serviço, uma vez que, custos logísticos tendem a aumentar com taxas crescentes à medida que nível de serviço é empurrado para níveis mais altos". Empresas que não se adequaram aos padrões de custos do seu segmento ou não implantaram controles de qualidade adequados "dificilmente conseguirão atuar de forma integrada e com sucesso na cadeia de suprimento otimizada" [16].

O planejamento estratégico logístico pode ser um dos caminhos para se atingir estes objetivos. O aumento da competitividade, clientes mais exigentes fazem com que as empresas sejam mais criativas, inovadoras e se diferenciem de seus concorrentes para que possam rentabilizar mais lucros. Muitas empresas estão buscando a excelência em logística para vencer estes desafios.

conhecimento das atividades pertinentes da empresa e também a todos os seus setores bem como as áreas que precisam de melhorias. A definição do escopo desta pesquisa, que se limitou ao processamento das perdas recorrentes de problemas no layout da empresa que acarreta na diminuição da capacidade produtiva e o tempo de entrega do produto ao cliente. 
De acordo com Yin, uma pesquisa empírica que investiga um fenômeno contemporâneo dentro de seu contexto na vida real, especialmente quando as fronteiras entre contexto e fenômeno não estão explicitamente evidentes. A pesquisa do estudo de caso beneficia-se então de um desenvolvimento de proposições teóricas anteriores para guiar a coleta e análise dos dados [17].

A primeira etapa foi encontrar o grau de congestionamento do tráfego dos serviços de transporte logístico dentro e fora da empresa, o principal objetivo desta etapa do estudo é a localização dos gargalos com previsões de fluxo de trafego em dias normais e anormais, nesta etapa foram realizada a coleta de dados a partir de entrevistas com colaboradores, checklist de observação direta, pesquisa documental. A entrevista é um encontro entre duas pessoas afim de que uma delas obtenha informações a respeito de determinado assunto, mediante uma conversação de natureza profissional. É um procedimento utilizado na investigação social para a coleta de dados ou para ajudar no diagnostico ou no tratamento de um problema social [3].

De acordo com [18], "na observação direta o pesquisador observa os fatos de maneira espontânea e atua como espectador". Segundo [19], a observação direta "[...] utiliza os sentidos na obtenção de determinados aspectos da realidade. Não consiste apenas em ver e ouvir, mas também examinar fatos ou fenômenos que se deseja estudar".

A pesquisa documental, devido a suas características, pode chegar a ser confundida com a pesquisa bibliográfica, a principal diferença entre esses tipos de pesquisa a natureza das fontes de ambas as pesquisas.

A bibliográfica utiliza-se principalmente das contribuições de vários autores sobre determinada temática de estudo, já a documental baseia-se em materiais que ainda não recebera um tratamento analítico ou que podem ser reelaborados de acordo com os objetivos da pesquisa [3].

A coleta e análise dos dados se referiram à distância entre a indústria e o cliente (em $\mathrm{km})$; o tempo de saída do caminhão do interior da empresa; o tempo do trajeto da empresa ao cliente (obra). Seguindo a análise e interpretação de dados que serão trabalhados para transformação dos mesmos em informações qualitativas e quantitativas relevantes para a pesquisa.

\section{APLICAÇÃO DO ESTUDO DE CASO IV.1. PERFIL DA EMPRESA}

A indústria de concreto usinado onde a pesquisa foi realizada se localiza na cidade de Manaus e fabrica seu produto de acordo com a necessidade dos clientes. Inserida num espaço físico de $21.000 \mathrm{~m}^{2}$, onde estão localizados os departamentos, administração, produção e circulação das máquinas e veículos que ocupam o maior espaço. Essa forma de circulação interna torna inviável parte do processo produtivo, fazendo com que surjam perdas significativas onerando os custos e consequentemente diminuindo a receita da organização.

A empresa Supermix S/A, na realização de suas tarefas diárias, vive esses momentos em seu processo produtivo, seja no abastecimento de insumos, ou na entrega de seu produto "concreto usinado".

Congestionamentos se formam dentro da organização. Caminhões se enfileiram em torno de uma única via de mão dupla, dividindo o espaço disponível com um trator, máquina usada para o abastecimento da linha de produção, as betoneiras carregadas de concreto, ou vazias aptas para um novo abastecimento, carros de clientes, fornecedores, funcionários e pedestres que trabalham e circulam em torno de toda a área.

O tema deste artigo é a logística, que pode ser definida de várias maneiras por diferentes estudiosos sobre o assunto, segundo [20], apresenta um conceito bastante abrangente, na qual é considerada como um processo de gerenciamento estratégico para a aquisição, a movimentação e a armazenagem de insumos, componentes e produtos acabados, incluindo os fluxos de informações correlatas. A intenção de identificar as deficiências quanto ao layout se fundamenta no argumento de [20], que se refere à infraestrutura física é responsável pelo inter-relacionamento dos interesses de uma organização e da comunidade, pois permite a elevação dos níveis de eficiência organizacional e oportuniza o atendimento de mercados mais amplos.

A problemática dar-se a partir do diagnóstico organizacional aplicado à empresa, no qual se pode notar a maior incidência de pontos fracos e pontos a melhorar na área de meio físico, ou seja, no layout da empresa.

\section{IV.2. APRESENTAÇÃO DOS DADOS DA ORGANIZAÇÃO}

Por meio de pesquisa de campo foram aplicadas técnicas de coleta de dados, foi feito um checklist de observação direta no qual foi possível analisar e tabular os dados coletados de forma qualitativa e quantitativa, após esse processo se deu a formulação de gráficos conforme figura 1, no qual observamos a estrutura e adequação dos setores, das vias de movimentação e das instalações da empresa pesquisada. 
Alfredo Nogueira Mariano, Emanoel C. Campos, Sandro O. Nunes/ITEGAM-JETIA Vol.01, Nº 04, pp.53-59. Dezembro, 2015.

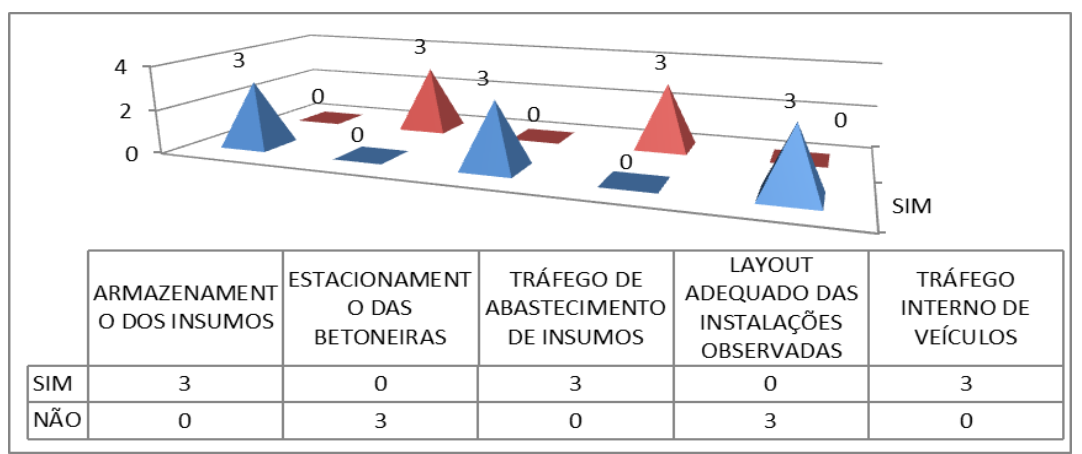

Figura 1 - Checklist.

Como podemos observar na figura, no setor de armazenamento de insumos obtivemos três votos sim o que comprova a sua adequação perante a observação direta dos pesquisadores, no item estacionamento das betoneiras temos três votos não, o que mostra a inadequação, pois os mesmos não existem, no item tráfego de abastecimento de insumos temos três votos sim o que nos mostra a verificação de constante movimentação na entrada de insumos na empresa, no item layout temos três votos de não adequação que implica em congestionamentos interno de máquinas e caminhões gerando perda na produção. No item tráfego interno de veículos temos três votos sim o nos mostra a grande movimentação no interior da organização como mostra a figura 2.

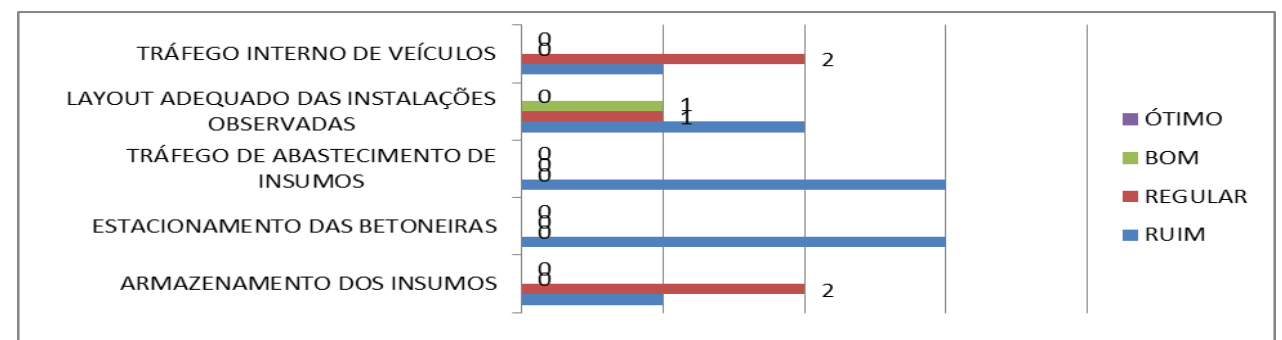

Figura 2-Checklist.

Nesta figura temos votos de qualitativos que variam de ótimo a ruim das adequações dos pontos observados a partir do chcklist. O problema logístico começa dentro da empresa, pelo fato do layout mal dimensionado, com pouco espaço para tráfego de caminhões que chegam a todo o momento para abastecer o setor de armazenamento de insumos e na saída de caminhões com produto final, como pode ser mostrados nas figuras $3 \mathrm{~A}, 3 \mathrm{~B}, 3 \mathrm{C}$ e $3 \mathrm{D}$.

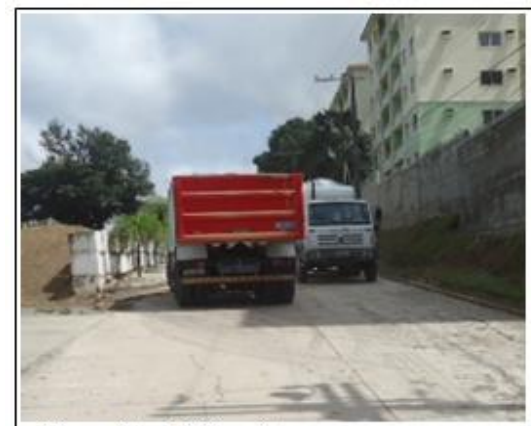

Figura 3A - Tráfego intemo.

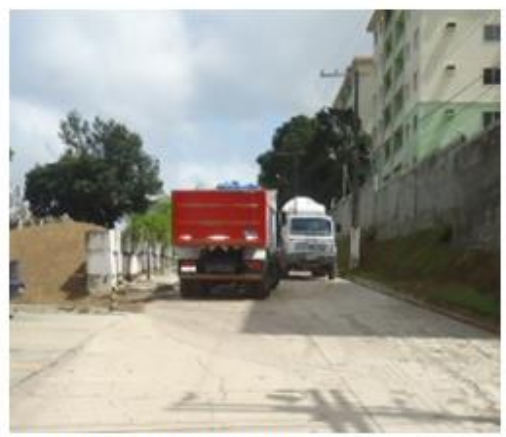

Figura 3C - Trá fego intemo.

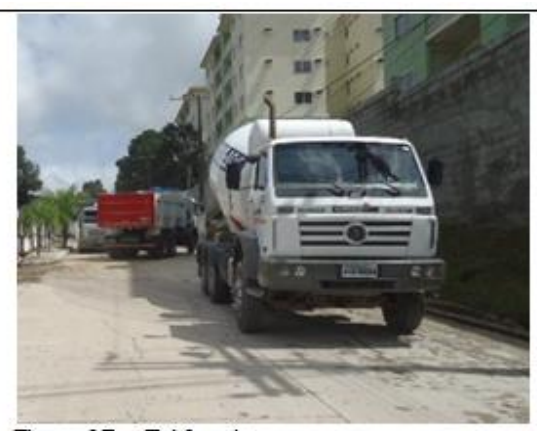

Figura 3B - Tráfego intemo.

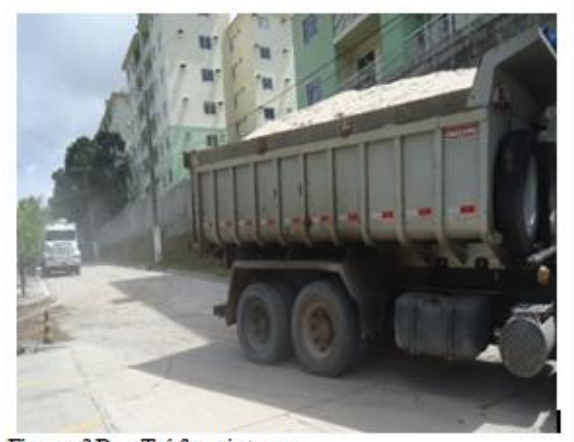

Figura 3D - Trá fego intemo

Figura 3 - Fluxo de movimentação da empresa. 
O transito de caminhões na entrada de insumos e saída de produto final, causa uma grande perda na produção diária, como pode ser observado nas figuras acima um caminhão de entrega tem que parar no passeio para que o outro possa manobrar várias vezes até o posicionamento adequado para despejo da matéria-prima no seu local de armazenamento, observa-se também a fila que se forma para o despejo do mesmo insumo que é um dos principais na formulação do produto final "concreto usinado", a parada e o tempo de espera no descarregamento dos insumos somados com outros agravantes como transito que nos seus horários de pico, esses problemas se transformam em uma grande perda de capacidade produtiva diária de um caminhão, como pode ser comprovado com um simples cálculo de capacidade de produção que é a quantidade máxima de produtos ou serviços que a empresa pode produzir em certo intervalo de tempo ou dia de trabalho. Existem vários tipos de Capacidade produtiva: Capacidade instalada pode ser traduzida como o limite da produção ou a capacidade máxima de produção de uma fábrica. É a quantidade de unidades de produto que as máquinas e equipamentos instalados são capazes de produzir; Capacidade disponível é o estudo de capacidade da produção sobre o tempo disponível do funcionamento da empresa. O calculo é bem simples também.
A fórmula é semelhante à da capacidade instalada, porém considera o tempo que a empresa trabalha. Vamos aos cálculos: 1 caminhão carregado a cada 15 minutos, ou seja, 4 por hora, porém esta fabrica trabalha em horário comercial: ou seja, das $08 \mathrm{~h} 00 \mathrm{~min}$ até as $17 \mathrm{~h} 00 \mathrm{~min}$. A solução do cálculo ficaria assim: 4 por hora $x 8$ horas de atividade da empresa $=$ 32 betoneiras cheias por dia. O mesmo cálculo foi feito exemplificando o carregamento (15 minutos), saída da empresa sem atraso, chegada até a obra que está aproximadamente 10 $\mathrm{km}$ de distância em (35 minutos), sem transito, descarga e volta para empresa, $\mathrm{C}=1$ caminhão carregado $x(15 \mathrm{~min}+35$ $\mathrm{min} / 60 \mathrm{~min}$ ) deslocamento $x 8$ horas disponíveis. $\mathrm{C}=1 \times 1,2 x$ $8=9,6$ caminhões carregados por dia.

Capacidade produtiva de um caminhão-betoneira sem atrasos. Também foi calculado esse mesmo percurso sofrendo interferências tanto com problemas dentro da empresa, como o atrasa na saída por conta da espera no descarregamento de insumos e atraso no transito. Carregamento (15 minutos), saída com atraso e transito em horário de pico (01 h $40 \mathrm{~min})$, descarga e volta para empresa. $\mathrm{C}=1$ caminhão carregado $x(15$ $\mathrm{min}+100 \mathrm{~min} / 60 \mathrm{~min}) \times 8$ horas disponíveis. $\mathrm{C}=1 \times 0,52 \times 8$ $=4,16$ caminhões carregados por dia. Capacidade produtiva de caminhão com atrasos. Após a execução desses cálculos foi possível mensurar esta perda e mostrá-la em forma de gráficos conforme figura 4 .

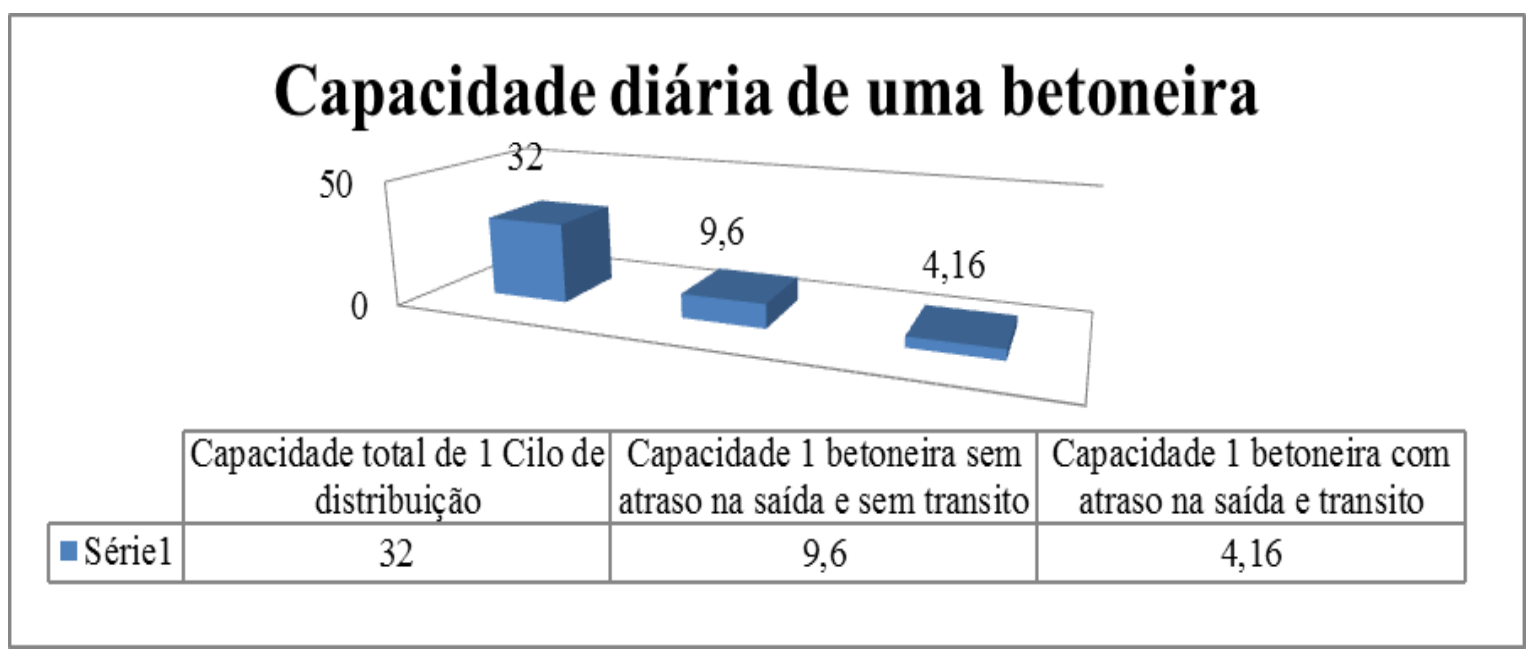

Figura 4 - Capacidade produtiva total e capacidade por caminhão com e sem atrasos.

A figura 4 mostra a capacidade total de um cilo com linha de produção, que tem a capacidade de encher 32 caminhões-betoneira por dia cerca de 256 metros cúbicos de concreto usinado. Capacidade total de uma betoneira em operação de entrega do concreto na obra sem atrasos e capacidade total de uma betoneira em operação de entra de concretos na obra com perdas. Os inúmeros congestionamentos gerados pelo fluxo de veículos em torno da capital refletem na inexatidão das entregas, perdas de materiais, falta de espaço para carga e descarga nas vias principais e secundárias, que se misturam com a logística de transporte de outras organizações e do PIM, são motivos concomitantes, a um processo de entrega tão pouco eficaz, em consequência, a falta de satisfação garantida. 


\section{IV.3. ANÁLISE, RESULTADOS E DISCUSSÕES DA PESQUISA}

As decisões de arranjo físico definem como a empresa vai produzir. O layout, ou arranjo físico é a parte mais visível e exposta de qualquer organização. A necessidade de estudá-lo existe, sempre que se pretende a implantação de uma nova fábrica, setor ou quando se estiver promovendo a reformulação de plantas industriais já em funcionamento.

O mal dimensionamento do layout é um dos problemas que tem influência direta na logística de transporte e distribuição do produto final da empresa, somados a outros fatores externos, como o transito caótico da cidade de Manaus afetam a capacidade produtiva e consequentemente na rentabilidade da organização. Após a análise e cálculos com os dados chegamos aos seguintes resultados.

A capacidade produtiva total de um ciclo com linha de produção é de 32 caminhões betoneira carregados por dia com 8 horas de trabalho. Considerando valor médio do $\mathrm{m}^{3}$ do concreto usinado que custa $\mathrm{R} \$ 400,00$ dependendo da sua composição, aplicação e aditivos, como o gelo que dá mais liga e resistência ao concreto. $\mathrm{O}$ balão de uma betoneira comporta 8 $\mathrm{m}^{3}$ de concreto. Esta quantidade expressada em valores, $8 \mathrm{~m}^{3} x$ $\mathrm{R} \$ 400,00=\mathrm{R} \$ 3.200,00 \times 32$ caminhões $=\mathrm{R} \$ 102.400,00$ por dia, ou seja, R\$ 2.457.600,00 por mês (24 dias) e R\$ 29.491.200,00 por ano. Calculando o valor médio do $\mathrm{m}^{3}(\mathrm{R} \$$ 400,00) $x$ a capacidade produtiva de um caminhão betoneira sem atrasos temos o seguinte resultado $8 \mathrm{~m}^{3} x \mathrm{R} \$ 400,00=\mathrm{R} \$$ 3.200,00 $x$ 9,6 caminhões $=\mathrm{R} \$ 30.720,00$ (trinta mil $\mathrm{e}$ setecentos e vinte reais) por dia, $\mathrm{R} \$ 737.280,00$ por mês e $\mathrm{R} \$$ 8.847.360,00 por ano.

O mesmo cálculo feito com os dados de um caminhão que sofreu atrasos na saída da empresa e congestionamentos nas vias da cidade, $8 \mathrm{~m}^{3} \times \mathrm{R} \$ 400,00=\mathrm{R} \$ 3.200,00 \times 4,16=$ $\mathrm{R} \$ 13.312,00$ por dia, $\mathrm{R} \$ 319.488,00$ por mês e $\mathrm{R} \$ 3.833 .856$ por ano. Pode-se observar uma perda produtiva superior a $50 \%$ na capacidade produtiva e na capacidade de entrega de um caminhão. Os objetivos da pesquisa fora alcançados, pois a partir dos dados podemos encontrar o ponto do gargalo, onde se ocasiona a perda e mensurar esta perda em valores em moeda corrente para que numa segunda etapa implantar um projeto para correção deste problema. A perda de capacidade produtiva de entrega, tendo como um dos fatores principais os problemas logísticos no interior e exterior da empresa causando estes que cooperam para a perda de lucros.

Por ser uma empresa multinacional de grande porte e ter como clientes as maiores construtoras do Brasil: Andrade Guiterrez, Capital Rossi, Aliança Construtora e ainda esta presente nas maiores obras na nossa capital amazonense como: Arena da Amazônia; Shopping Ponta Negra; Shoping Via Norte entre outras, com os dados obtidos pode-se dar inicio em uma tomada de ação para a correção do erro e corrigir esta patologia na organização por meio de uma reformulação da planta industrial, alterando as vias da empresa para que se tenha uma melhora no escoamento do produto final até o seu consumidor.

\section{CONCLUSÕES}

A partir dos dados levantados e dos resultados apresentados no processo de verificação do problema, pode-se concluir que os objetivos desta pesquisa foram alcançados, pois a partir dos dados coletados podemos encontrar o ponto do gargalo, onde e como se ocasiona está perda e calculá-la em capacidade produtiva e em valores em moeda corrente assim chegando ao resultado esperado, mostrando de forma clara com cálculos de produção e gráficos que possibilitam uma melhor visualização das perdas dentro da organização.

As consequências do mau dimensionamento do layout, que por sua vez implica nos problemas logísticos da empresa. Pode-se concluir que é necessário fazer um estudo de qual tipo de layout adequado para cada tipo de organização, pois o arranjo físico define como a empresa vai produzir, assim evitando problemas na produção e na movimentação interna, que pode causar outros problemas, como o exposto neste artigo os problemas logísticos. A importância deste estudo para o avanço da pesquisa de gestão organizacional é relatar o processo logístico e administração que se faz necessário nas organizações tendo como base à empresa Supermix Concretos S/A, a logística é fundamental para o sucesso das organizações.

\section{AGRADECIMENTOS:}

Ao Centro Universitário do Norte (UNINORTE), pelo apoio a realização desta pesquisa, a empresa utilizada na pesquisa SUPERMIX CONCRETO S/A.

\section{REFERÊNCIAS BIBLIOGRÁFICAS}

[1] BALlOU, R.H. Gerenciamento da Cadeia de Suprimentos. $1^{\circ}$ ed. Porto Alegre, 1993.

[2] CHRISTOPHER, M. Logística e gerenciamento da cadeia de suprimento: estratégias para. A redução de custo e melhoria dos serviços. São Paulo: Pioneira, 2002.

[3] LAKATOS, Eva Maria; MARCONI, Marina de Andrade. Fundamentos de Metodologia Científica. $6^{\mathrm{a}}$. Ed. São Paulo: Atlas, 2005.

[4] RODRIGUES, J. P. Rodrigues. Globalization and the Synchronization of Transport Terminals. Journal of Transport Geography, 7 (1999), pp. 255-261

[5] SLACK, Nigel; CHAMBERS, Stuart; JOHNSTON, Robert. Administração da Produção. São Paulo: Atlas, 2002. pp. 396-413.

[6] DORING, Rubins. Desenvolvimento gerencial. São Paulo: Fundação Getúlio Vargas, 1998.

[7] BRAUDEL, F. Braudel. As rodas de Comércio, Civilização e Capitalismo 150180 século. Vol. II, Harper e Row, Nova Iorque, 1982.

[8] DICKENS e THRIFT, $\mathrm{P}$ Dickens, $\mathrm{N}$ Thrift. A organização da produção e a produção de organização: por que as empresas importam no estudo da industrialização geográfica Transações do Instituto de Geógrafos britânicos, 17 (1992), PP. 270-291.

[9] GERTLER, M. Flexibilidade revisitada: distritos, os estados - nação, e as forças de produção Transações do Instituto de Geógrafos britânicos, 17 (1992), PP. 259-278.

[10] HUDSON, R. Hudson. Producing Places Guilford, London (2001). 
[11] HANDFIELD and NICHOLS, R.B Handfield, E.L Nich Introduction to Supply Chin Management. 1999.

[12] BALlOU, R.H. Gerenciamento da Cadeia de Suprimentos - Logística Empresarial. 5. ed. Porto Alegre: Bookman, 2006. $616 \mathrm{p}$.

[13] BOWERSOX et al. D. Bowersox, Closs D, T Stank, Dez mega-tendências que irão revolucionar a log ística da cadeia de abastecimento. Jornal de Negóci os de Logística, 21 (2000), PP 1-16.

[14] FERREIRA, Aurélio Buarque De Holanda. Novo Dicionário Aurélio da Língua Portuguesa. $2^{\mathrm{a}}$ ed. Rio de Janeiro: Nova Fronteira, 1986.

[15] COUNCIL OF SUPPLY CHAIN MANAGEMENT PROFESSIONALS - CSCMP. Supply Chain - Logistics Definition. Disponívelem: <http: //cscmp.org/AboutCSCMP/ Definitions/Definitions.asp>. Acesso em: 01 abril. 2014.

[16] NOVAES, Antônio Galvão. Logística e gerenciamento da cadeia dedistribuição $-2^{\circ} \mathrm{ed}$. Rio de Janeiro; Elsevier, 2007.

[17] YIN, R.K., "Case Study Research: desing and methods". $2^{\mathrm{a}}$ ed. Applied social research methods series; v.5, London: SAGE Publications, 2010.

[18] GIL, Antônio Carlos. Como elaborar projetos de pesquisa. 3a.ed. São Paulo: Altas. 1991.158 p.

[19] GIL, Antônio Carlos. Como elaborar projetos de pesquisa. $4^{\circ}$ Ed. São Paulo: Atlas, 2007.

[20] MUSKIN, J. B. The physical distribuition infrastructure. Transportation Quartely, Westpon: Eno Fundation for Transportation, v. 37, n. 1, p. 115-133, Jan. 1983. 